[Case Report]

\title{
Pembrolizumab-induced Radiation Recall Pneumonitis After the Resolution of Typical Asymptomatic Radiation Pneumonitis
}

\author{
Hirohide Itamura $^{1}$, Takayuki Ohguri ${ }^{1 *}$, Katsuya Yahara $^{1}$, Sota NAKahara ${ }^{1}$, Sho KaKinouchi, \\ Takahiro Morisaki $^{1}$, Kazuhiro YATERA ${ }^{2}$, Fumihiro TANAKA ${ }^{3}$ and Yukunori Korogi ${ }^{1}$ \\ ${ }^{1}$ Department of Radiology, School of Medicine, University of Occupational and Environmental Health, Japan. \\ Kitakyushu 807-8555, Japan \\ 2 Department of Respiratory Medicine, School of Medicine, University of Occupational and Environmental Health, \\ Japan. Kitakyushu 807-8555, Japan \\ ${ }^{3}$ Second Department of Surgery, School of Medicine, University of Occupational and Environmental Health, Japan. \\ Kitakyushu 807-8555, Japan
}

\begin{abstract}
Radiation recall pneumonitis is a phenomenon in which a recall-triggering drug induces an acute inflammatory reaction in the lungs, corresponding to a previously irradiated area. Radiation recall reactions have been reported to occur following treatments with various cytotoxic anticancer agents and molecular-targeting drugs; however, only a few reports have described immune checkpoint inhibitor-induced radiation recall pneumonitis. We report a case of radiation recall pneumonitis induced by pembrolizumab in a patient with the postoperative local recurrence of non-small cell lung cancer. This case demonstrated that pembrolizumab might cause severe radiation recall pneumonitis, even after typical radiation pneumonitis has been resolved.
\end{abstract}

Keywords : pembrolizumab, non-small cell lung cancer, radiation pneumonitis, radiotherapy, radiation recall reaction.

(Received May 7, 2020, accepted July 7, 2020)

\section{Introduction}

Radiation recall reaction is a phenomenon in which a recall-triggering drug induces an acute inflammatory reaction in a site corresponding to a previously irradiated area. Typically, chemotherapy-induced dermatitis appears in the skin corresponding to previously irradiated areas after the administration of a chemotherapeutic agent. The most common site for radiation recall reactions is the skin, but these reactions can also occur in the lungs, mucosa, gastrointestinal tract, and central nervous system [1]. The development of radiation recall reactions has been reported in response to many anticancer drugs [1-5].

Immune checkpoint inhibitors have rapidly been introduced as cancer treatments in recent years. A cancer-specific immunostimulatory effect, induced by radiation therapy, has also been reported, and the combination of radiation therapy and immunotherapy has received increasing interest. We report a case of severe radiation recall pneumonitis, induced by pembrolizumab, an immune checkpoint inhibitor (ICI) that acts as an anti-programmed death receptor-1 (PD-1) drug, after the resolution of typical asymptomatic radiation pneumonitis.

*Corresponding Author: Takayuki OHGURI, Department of Radiology, University of Occupational and Environmental Health, Japan. Yahatanishi-ku, Kitakyushu 807-8555, Japan, Tel: +81-93-691-7264, Fax: +81-93-692-0249, E-mail: ogurieye@med.uoeh-u.ac.jp 


\section{Case Report}

The patient had undergone a left upper lobectomy and lymph node dissection for stage I non-small cell lung cancer eight years and nine months prior to our treatment. Although neither recurrence nor metastasis was observed, new lung cancer was found in the right lower lobe 3 years and 2 months previously, and the excision of right lower lobe segments ( $\mathrm{S} 6-\mathrm{S} 8$ ) was performed. The postoperative pathological diagnosis was adenocarcinoma (pT1N0M0). No mutations were identified in the epidermal growth factor receptor gene, anaplastic lymphoma kinase fusion gene, or ROS1 fusion gene. The programmed death-ligand 1 (PD-L1) expression was $50-60 \%$. One year and 7 months before the time of the present report, computed tomography (CT) showed an increase in the resected margin soft tissue of the right lower lobe of the lung and the enlargement of the right hilar lymph node (Figure 1A). Local recurrence was diagnosed, based on high positron emission tomography (PET)/CT uptake [maximum standardized uptake value (SUVmax) of 14.26], and salvage chemoradiotherapy was performed.

For radiotherapy, a total dose of $64 \mathrm{~Gy}$, in 32 fractions, was administered to the recurrent lesions, based on 3-dimensional treatment planning, and prophylactic irradiation of 40 Gy was administered to the right hilar and upper mediastinal lymph nodes (Figure 1B). The dose parameters were a lung V20 of $25.9 \%$, a lung V5 of 38\%, and a mean lung dose of $15 \mathrm{~Gy}$, which are the standard dosing regimens for postoperative recurrent lung cancer. Concurrent chemotherapy consisted of weekly administration of carboplatin (area under the curve of 2) and paclitaxel $\left(40 \mathrm{mg} / \mathrm{m}^{2}\right)$.

$\mathrm{CT}$ performed 2 months after the completion of radiotherapy showed a complete response in the recurrent lesion. Three months after the completion of radiotherapy, CT showed the appearance of infiltrative shadows, which were well-matched with the irradiated high-dose area, with no subjective symptoms (Figure $2 \mathrm{~A}$ ). We diagnosed the shadow as grade 1 typical radiation pneumonitis, based on Common terminology criteria for adverse events, version 5.0.

Multiple bone metastasis, bilateral adrenal metastasis, and abdominal para-aortic lymph node metastasis were observed at 4 months after the completion of radiotherapy. The administrations of tri-weekly carboplatin (area under the curve of 4) and weekly nabpaclitaxel $\left(100 \mathrm{mg} / \mathrm{m}^{2}\right)$ were started. CT at 5 months after the completion of radiotherapy showed that the radiation pneumonitis had resolved spontaneously (Figure 2B).

Brain metastases were recognized in magnetic resonance imaging scans at 6 months after the completion

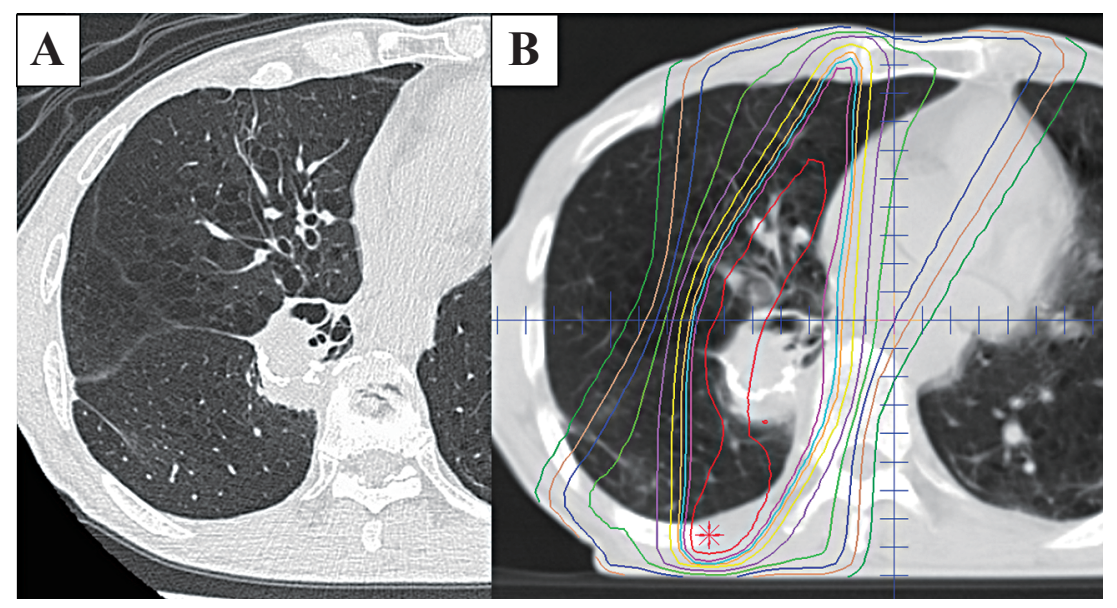

Figure 1. Radiotherapy for postoperative local recurrence. A: Computed tomography $(\mathrm{CT})$ imaging of postoperative local recurrence in the patient with non-small cell lung cancer. B: Dose distribution of radiotherapy administered for the treatment of postoperative local recurrence. Red line: 66 Gy, Magenta line: 64 Gy, Yellow line: 56 Gy, Blue line: 30 Gy, Dark green line: 20 Gy. 
of radiotherapy. Pembrolizumab $(4.5 \mathrm{mg} / \mathrm{kg})$ monotherapy was initiated as a new systemic treatment. Thirty-five days after the initial administration of pembrolizumab, the patient noted shortness of breath on exertion, and oxygen saturation $(\mathrm{SpO} 2)$ decreased to $93 \%$ (room air), requiring oxygen administration. CT showed recurrent pneumonia, corresponding to the mid- to high-dose area of the radiation field (Figure $3 \mathrm{~A})$. We diagnosed the patient as having radiation recall pneumonitis, induced by pembrolizumab, based on the course of treatment (Figure 4), CT findings, and the results of peripheral blood and bronchoalveolar lavage fluid tests, which were all consistent with radiation recall pneumonitis (Table 1). Pembrolizumab was discontinued, and 3 days of steroid pulse therapy (methylprednisolone at $1,000 \mathrm{mg}$ /day) was immedi- ately administered. Subsequently, the intravenous injection of water-soluble prednisolone $(60 \mathrm{mg} /$ day $)$ was administrated and the patient was switched to the oral administration of prednisolone ( $40 \mathrm{mg} /$ day). The symptoms rapidly improved, and 3 months after the initiation of steroid therapy, CT showed a marked improvement of the radiation recall pneumonitis (Figure 3B). Prednisolone was tapered off over 8 months and then discontinued. No recurrence of radiation recall pneumonitis was recognized.

Subsequently, palliative radiotherapy was performed for multiple bone metastases, and stereotactic radiosurgery was performed for brain metastases, but the multiple metastases progressed, and the patient died 2 years and 1 month after salvage chemoradiotherapy for postoperative recurrent lung cancer.

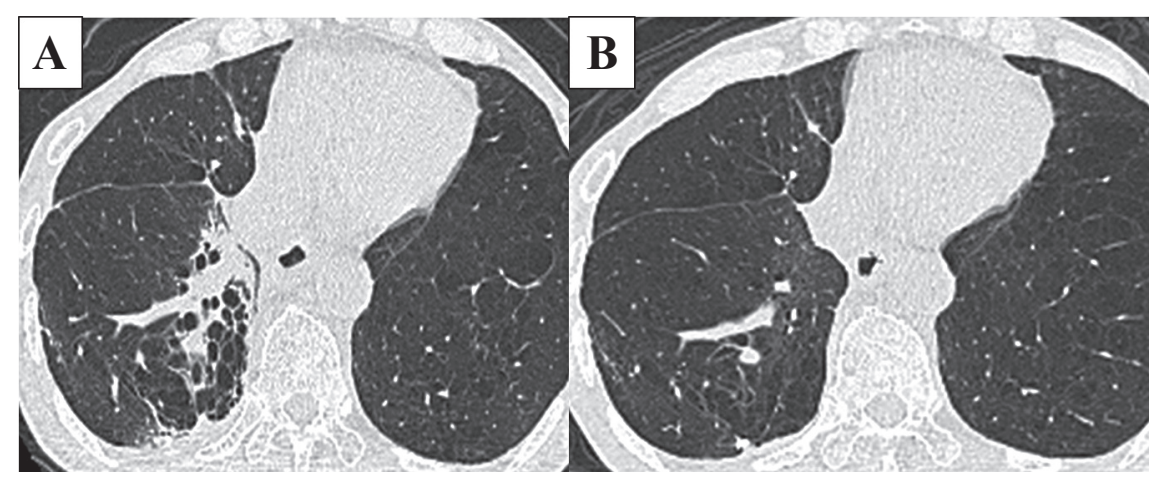

Figure 2. Asymptomatic radiation pneumonitis. A: Asymptomatic radiation pneumonitis was recognized three months after the completion of radiotherapy, corresponding to the previously irradiated area. B: The radiation pneumonitis resolved spontaneously 2 months later.

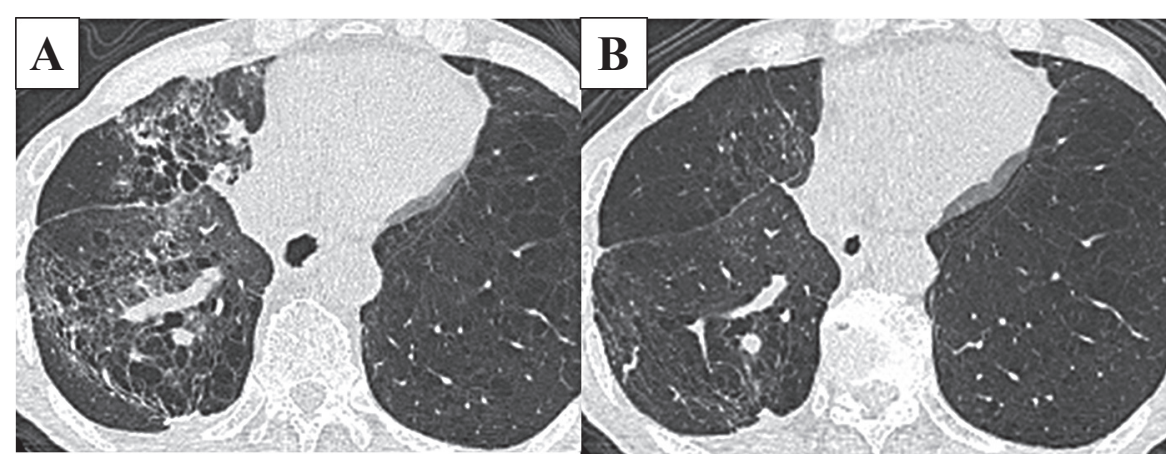

Figure 3. The clinical course of radiation recall pneumonitis. A: Computed tomography (CT) imaging at the onset of radiation recall pneumonitis showed the appearance of widespread pneumonia corresponding to the area receiving middle- to high-dose radiation in the right lung, with pulmonary emphysema. B: Radiation recall pneumonitis had improved three months after the initiation of steroid therapy. 


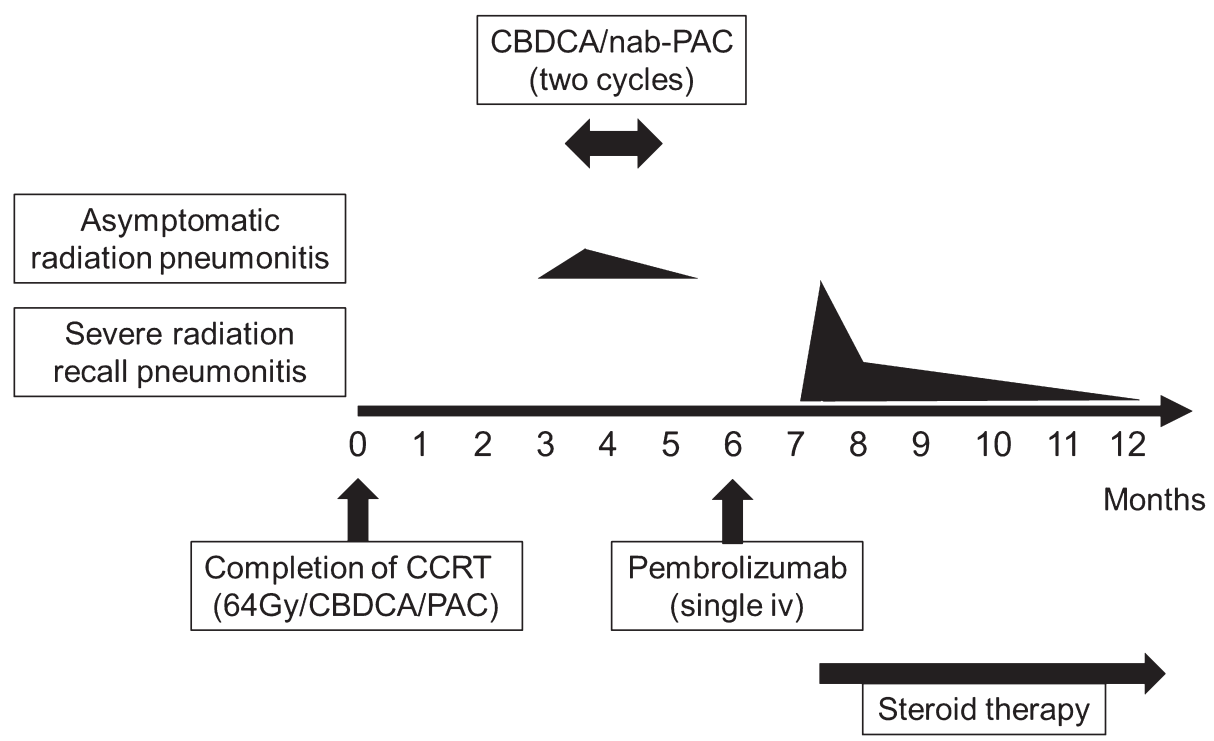

Figure 4. Overview of the time course and treatment of the disease. CBDCA: carboplatin, nab-PAC: nab-paclitaxel, CCRT: concurrent chemoradiotherapy, PAC: paclitaxel, iv: intravenous infusion.

Table 1. Laboratory findings at the onset of radiation recall pneumonitis

\begin{tabular}{|c|c|}
\hline Clinical test items & \\
\hline \multicolumn{2}{|l|}{ Peripheral blood test findings } \\
\hline $\operatorname{WBC}(/ \mu l)$ & $8.5 \times 10^{3}$ \\
\hline NEUTRO (\%) & 80.7 \\
\hline LYMPH (\%) & 6.2 \\
\hline EOS $(\%)$ & 2.5 \\
\hline MONO (\%) & 10.5 \\
\hline $\operatorname{HGB}(\mathrm{g} / \mathrm{d} l)$ & 10.7 \\
\hline $\operatorname{PLT}(/ \mu l)$ & $211 \times 10^{3}$ \\
\hline $\mathrm{CRP}(\mathrm{mg} / \mathrm{d} l)$ & 0.64 \\
\hline $\mathrm{KL}-6(\mathrm{U} / \mathrm{m} l)$ & 2,101 \\
\hline \multicolumn{2}{|l|}{ Bronchoalveolar lavage fluid } \\
\hline Puncture fluid cell count $(/ \mu l)$ & 50 \\
\hline Neutrophil (\%) & 17 \\
\hline Lymphocyte (\%) & 10 \\
\hline Eosinophile (\%) & 6 \\
\hline Macrophages (\%) & 67 \\
\hline CD4 (\%) & 42 \\
\hline CD8 (\%) & 48 \\
\hline $\mathrm{CD} 4 / \mathrm{CD} 8$ & 0.88 \\
\hline
\end{tabular}

WBC: White blood cell, NEUTRO: Neutrophil, LYMPH: Lymphocyte, EOS: Eosinophils, MONO: Mononucleosis, HGB: Hemoglobin, PLT: Platelet count, CD: Cluster of differentiation

\section{Discussion}

The present case is remarkable because it demonstrated that pembrolizumab-induced radiation recall pneumonitis can occur, even after asymptomatic radiation pneumonitis has been resolved, as the severe relapse of radiation pneumonitis appeared to be induced by pembrolizumab administration.

Radiation recall reaction has been demonstrated to occur with the administration of cytotoxic anticancer agents such as methotrexate, paclitaxel, docetaxel, and gemcitabine, as well as molecular-targeting drugs such as trastuzumab, gefitinib, and osimertinib [2-4, 6-9]. Although the pathogenesis of radiation recall reaction is not understood in detail, Kitani et al reported that the cells that remain following radiation therapy may be intolerant to chemotherapy, due to a decrease in the number of tissue stem cells and genetic mutations [10].

For ICIs, 2 cases of radiation recall pneumonitis have been reported for nivolumab, an anti-PD-L1 drug [11]. Both cases received definitive radiation therapy, at $60 \mathrm{~Gy}, 2$ years before the onset of radiation recall pneumonitis, with the appearance of pneumonia corresponding to the irradiated area. Both of those patients were treated with oral steroids, and their symptoms rapidly improved. To our knowledge, only one brief 
description of a patient with pembrolizumab-induced radiation recall pneumonitis has been reported [12]. The reported patient experienced asymptomatic radiation recall pneumonitis 10 months after the administration of pembrolizumab, which corresponded to the area that had been irradiated 14 months earlier. Prednisone, at $1 \mathrm{mg} / \mathrm{kg}$, was initiated, followed by a prolonged tapering off. Repeated CT imaging 3 months later showed the resolution of the radiation recall pneumonitis shadow.

In our case, the typical, asymptomatic (Grade 1) radiation pneumonitis that occurred 3 months after the completion of irradiation was resolved. However, 6 months after the completion of irradiation, the patient presented with severe symptomatic pneumonia 35 days after the administration of pembrolizumab, and the pneumonia progressed rapidly. In previous reports of radiation recall reaction in response to cytotoxic anticancer drugs, the time from completion of irradiation to the development of radiation recall reaction ranged from 2 days to 15 years. The median time from the first administration of cytotoxic anticancer drugs to the onset of radiation recall pneumonitis was 42 days [5]. Previous reports of radiation recall pneumonitis have also described good responses to steroid therapy. Although no consensus exists regarding whether steroid pulse therapy is necessary for radiation recall pneumonitis, selection of steroid pulse therapy should based on the severity of the disease. We diagnosed the pneumonia in our case as radiation recall pneumonitis due to the course of treatment, the good response to steroids, and the consistent peripheral blood and bronchoalveolar lavage fluid test results.

Pembrolizumab is an anti-PD-1 ICI that has been used as monotherapy and in combination with other anticancer drugs to treat unresectable, advanced, or recurrent non-small cell lung cancer. ICIs can cause inflammatory reactions, termed immune-related adverse events (irAEs), such as endocrine disorders, skin reactions, colitis, and pneumonia [13, 14]. The occurrence of immune-related pneumonitis following the administration of pembrolizumab has been reported to be approximately 3\% [15]. Su et al conducted a meta-analysis to determine the risks of pneumonia associated with various ICIs when treating solid tumors, and demonstrated that only pembrolizumab showed a significant increase in grade 3-5 pneumonitis, compared with chemotherapy [15]. ICI-induced pneumonia is associated with the dysregulation of effector and regulatory $\mathrm{T}$ cells in the lung interstitium, ultimately causing an inflammatory response [15]. Both PD-L1 and PD-L2 are ligands for PD-1. PD-L1 inhibitors do not inhibit the interaction between PD-L2 and PD-1; thus, PD-1 inhibitors may cause severe irAE compared with PD-L1 inhibitors. Xiao et al demonstrated that blocking the interaction between PD-L2 and repulsive guidance molecule $\mathrm{b}$ (RGMb), which is expressed by lung interstitial macrophages and alveolar cells, markedly impaired the development of respiratory tolerance [16].

Our experience with this case suggests that pembrolizumab may induce severe radiation recall pneumonitis, even after the resolution of typical asymptomatic radiation pneumonitis. The synergistic effects of ICIs and radiation therapy on the activation of antitumor immunity, including the "abscopal effect", have attracted attention, as ICIs are likely to be increasingly administered to patients who have received radiation. Recently, durvalumab, an anti-PD-L1 antibody, has been used during maintenance therapy after radical chemoradiotherapy in patients with unresectable, locally advanced, non-small cell lung cancer. There is a need for further reports and studies examining radiation recall pneumonitis caused by radiotherapy and ICIs.

\section{Conflict of Interest}

The authors declare no conflict of interest.

\section{References}

1. Schweitzer VG, Juillard GJ, Bajada CL \& Parker RG (1995): Radiation recall dermatitis and pneumonitis in a patient treated with paclitaxel. Cancer 76(6): 10691072

2. Burstein HJ (2000): Side effects of chemotherapy. Case 1. Radiation recall dermatitis from gemcitabine. J Clin Oncol 18(3): 693-694

3. Camidge DR (2001): Methotrexate-induced radiation recall. Am J Clin Oncol 24(2): 211-213

4. Morkas M, Fleming D \& Hahl M (2002): Challenges in oncology. Case 2. Radiation recall associated with 
docetaxel. J Clin Oncol 20(3): 867-869

5. Ding X, Ji W, Li J, Zhang X \& Wang L (2011): Radiation recall pneumonitis induced by chemotherapy after thoracic radiotherapy for lung cancer. Radiat Oncol 6: 24

6. McCarty MJ, Peake MF, Lillis P \& Vukelja SJ (1996): Paclitaxel-induced radiation recall dermatitis. Med Pediatr Oncol 27(3): 185-186

7. Moon D, Koo JS, Suh CO, Yoon CY, Bae J \& Lee S (2016): Radiation recall dermatitis induced by trastuzumab. Breast Cancer 23(1): 159-163

8. Inomata M, Miya T \& Kokubo Y (2010): Radiation recall pneumonitis induced by gefitinib in a patient with lung adenocarcinoma and EGFR mutation. Japanese Journal of Lung Cancer 50: 938-941

9. Sanchis-Borja M, Parrot A, Sroussi D, Rivin Del Campo E, Fallet V \& Cadranel J (2019): Dramatic radiation recall pneumonitis induced by osimertinib after palliative thoracic radiotherapy for lung cancer. J Thorac Oncol 14(10): e224-e226

10. Kitani H, Kosaka T, Fujihara T, Lindquist K \& Elkind MM (1990): The "recall effect" in radiotherapy: is subeffective, reparable damage involved? Int J Radiat Oncol Biol Phys 18(3): 689-695

11. Shibaki R, Akamatsu H, Fujimoto M, Koh Y \& Yamamoto N (2017): Nivolumab induced radiation recall pneumo- nitis after two years of radiotherapy. Ann Oncol 28(6): 1404-1405

12. McGovern K, Ghaly M, Esposito M, Barnaby K \& Seetharamu N (2019): Radiation recall pneumonitis in the setting of immunotherapy and radiation: a focused review. Future Sci OA 5(5): FSO378

13. Su Q, Zhang X, Shen X, Hou Y, Sun Z \& Gao ZH (2018): Risk of immune-related colitis with PD-1/PDL1 inhibitors vs chemotherapy in solid tumors: systems assessment. J Cancer 9(9): 1614-1622

14. Su Q, Zhang XC, Wang DY et al (2018):The risk of immune-related endocrine disorders associated with antiPD-1 inhibitors therapy for solid tumors: A systematic review and meta-analysis. Int Immunopharmacol 59: $328-338$

15. Su Q, Zhu EC, Wu JB et al (2019): Risk of pneumonitis and pneumonia associated with immune checkpoint inhibitors for solid tumors: A systematic review and meta-analysis. Front Immunol 10: 108

16. Xiao Y, Yu S, Zhu B et al (2014): RGMb is a novel binding partner for PD-L2 and its engagement with PD-L2 promotes respiratory tolerance. J Exp Med 211(5): 943-959

J UOEH $42(3): 261-266(2020)$ 